\title{
Correlative Fluorescence and Electron Microscopy in 3D
}

\author{
Donna Beer-Stolz ${ }^{1}$, Jonathan Franks ${ }^{1}$, Callen Wallace ${ }^{1}$, Masateru Shibata ${ }^{2}$, Mitsuo Suga ${ }^{3}$, Natasha \\ Erdman $^{2}$ and Simon Watkins ${ }^{1}$ \\ ${ }^{1}$ Center for Biologic Imaging, University of Pittsburgh \\ 2 JEOL USA Inc, Peabody, Massachusetts \\ ${ }^{3}$ JEOL Ltd., Akishima, Tokyo, Japan
}

The ability to correlate fluorescence microscopy (FM) and electron microscopy (EM) data obtained on biological (cell and tissue) specimens is essential to bridge the resolution gap between the data obtained by these different imaging techniques. In the past such correlations were limited to either EM navigation in 2D to the locations previously highlighted by fluorescence markers, or subsequent high resolution acquisition of tomographic information using a TEM [1]. While fluorescence microscopy can provide insight into location, interactions and transport of chemical species in biological specimens by utilizing fluorescent markers for imaging, the ultimate resolution of the technique is still limited to $\sim 200 \mathrm{~nm}$ range using standard approaches and between 20 and 120nm using super-resolution methods. The electron microscopy techniques (SEM and TEM) can provide the next step in resolution (in sub $1 \mathrm{~nm}$ regime) to help understand the interactions at the cellular level. The TEM, however, suffers from a relatively small field of view - just 1-2 mm.

In this paper we will present a comparative analysis of multiple CLEM methods, including live cell imaging of human immunodeficiency virus infection followed by cryo EM and FIB milling, as well as large area multicolor 3D confocal imaging followed by plastic embedment, polishing and SEM or sectioning and TEM. All approaches rely critically on the ability to cross correlate positions in 3D space between the light microscopy and EM data, discussion as to how this is achieved is a focus of the presentation.

The cryoEM approach is a method that we have been developing for the last few years and have published previously [2]. While it is useful is not generally accessible to imaging facilities that have standard confocal and EM (SEM and TEM facilities).

The second and entirely novel approach solves this dilemma and uses correlative mapping from 3D confocal microscopy to guide subsequent EM analyses. Importantly the approach is very effective for finding very rare events within very large areas $(12 \mathrm{~mm}$ circular areas of confluent cells). The light microscope allows us to map very large 3D volumes at the diffraction limit. Subsequently the cells are fixed, counterstained, embedded and processed with a sequential mechanical polish and scanning electron microscope imaging procedure to provide a 3D EM tomogram of the sample with direct correlation to the confocal data. The method utilizes a multi-step controlled mechanical polishing of the previously OM examined embedded specimen block followed by high-resolution SEM imaging at each polishing step. In each case the chosen locale investigated by EM is guided by the high resolution FM data. The resulting SEM images can be rendered as a 3D tomogram of the cells such that we can truly address "needle" in a haystack questions and localize protein interactions and define EM resolution images of the interactions between structure, or examine viral structure and position again with EM resolution but guided by the light microscope (Fig. 1). 
We will present a "guided tour" on how to build this protocol into your own facility environment and define how to correlate objects exactly within 3D space such that structures can be isolated exactly and reproducibly. We will also discuss the problems that may be met implementing the approach and give multiple examples of how the method works in practice.

\section{References.}

[1] Zhang, P. 2013 Curr. Opin. Struct. Biol. 23:763-770

[2] Hansen, S.D. et al. 2013. Molecular biology of the cell, 24(23), pp.3710-3720.
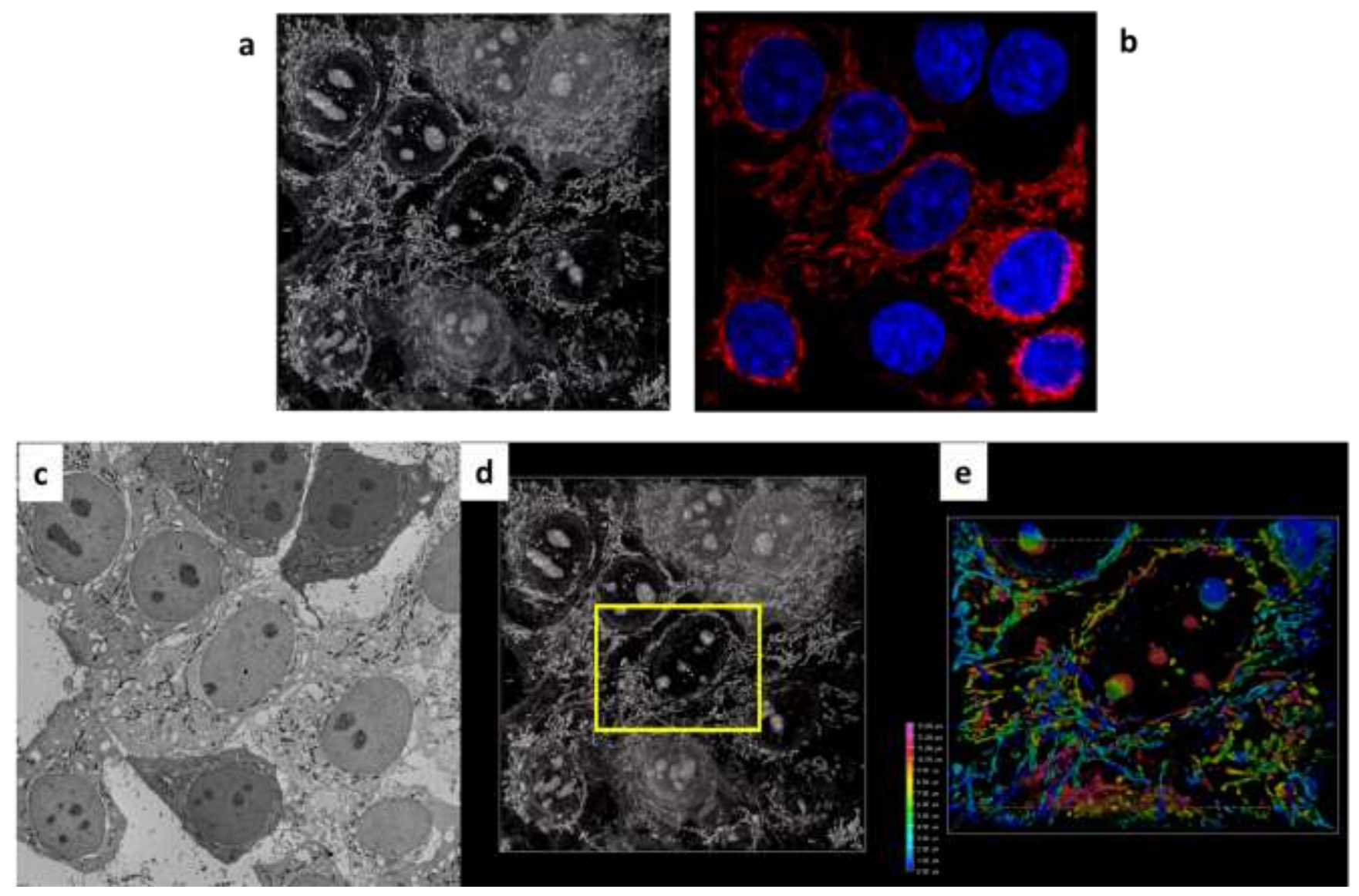

Fig. 1. a) Correlative 3D z-stacks of SEM (inverted to show cell structures (white)); b) FM images showing Tom20 (red) and nuclei (blue); c) Single plane SEM image; d) 3D view of reconstructed SEM images, inverted and skewed to highlight mitochondrial structure; e) Depth coded region of interest from image d. 